\title{
Género y conductas ansiosas en estudiantes universitarios*
}

\section{Gender and anxious behavior in university students}

Roxana Yolanda Castillo Acobo* ORCID: 000-0002-6467-7796

\section{Andrés Luque Ruiz de Somocurcio} ORCID: 0000-0002-7250-1221

Universidad Nacional de San Agustín de Arequipa, Perú

Recibido: 17 de mayo de 2018 Revisado: 26 de junio de 2018 Aceptado: 4 de agosto de 2018

\section{Resumen}

Las diferencias de oportunidades de género en el campo de la ciencia y tecnología son aún una brecha, a esto se suma las probables afectaciones psicológicas, emocionales que son de interés en el estudio porque analiza y relaciona de forma objetiva las implicaciones de género y riesgos por predisposición a conductas ansiosas en estudiantes de ciencia y tecnología de la universidad. Los participantes fueron de 4to. y 5to. año, de diferentes especialidades, la muestra estuvo constituida por 368 (192 mujeres y 176 hombres), que realizan actividades de investigación. Se aplicaron el cuestionario sobre Implicaciones de género y la escala del IDARE (Inventario de Ansiedad Rasgo-Estado) para medir ansiedad. Los resultados ponen de manifiesto que las estudiantes participan más en actividades de investigación pero también obtienen mayores niveles de ansiedad "estado". Se evaluó la vida familiar, el vivir solos, con familiares, tareas dentro del hogar,

Artículo de investigación. Citar como: Castillo, A. R. \& Luque R. de S. A. (2019). Género y conductas ansiosas en estudiantes universitarios. Diversitas: Perspectivas en Psicología, 15(1), 37-47. DOI: https://doi.org/10.15332/s1794-9998.2019.0001.03

Financiación: El presente trabajo es parte de una investigación que ha sido financiado con fondos del canon minero asignados a la Universidad Nacional de San Agustín de Arequipa, previo concurso, evaluación y selección de proyectos de investigación, en la cohorte 2016-2018.

Correspondencia: Dra. Roxana Castillo Acobo, Directora, Instituto de Investigaciones Sociales - UNSA, Perú. Correo electrónico: rcastilloa@ unsa.edu.pe. Dirección postal: Urb. Quinta Santa María N 13/ José Luis Bustamante y Rivero Arequipa-Perú. 
participación y aportes al conocimiento científico. Los estudiantes muestran mayor ansiedad "estado" al participar en círculos de estudio, por ser parte de investigaciones financiadas, frente a la paternidad y por responsabilidad económica en el hogar. Resaltar que hay claros indicios de afectaciones (ansiedad) a la salud psicológica-emocional por segregación vertical y horizontal de género.

Palabras Clave: género, ansiedad: Estado-Rasgo.

\section{Abstract}

The differences in gender opportunities in the field of science and technology are still a gap, in addition to the probable psychological and emotional effects that are of interest in the study because it analyzes and objectively relates the implications of gender and risks for predisposition to anxious behavior in university science and technology students. The participants are 4th and 5th year, of different specialties, the sample was constituted by 368 (192 women and 176 men), who carry out research activities. Methods: Two instruments were applied: a questionnaire on Gender Implications and the IDARE scale to measure anxiety. Results show that students participate more in research activities but also obtain higher levels of "state" anxiety. Family life, living alone, with relatives, tasks within the home, participation and contributions to scientific knowledge were evaluated. Students show greater anxiety "status" when participating in study circles, as part of funded research, as opposed to paternity and economic responsibility in the home. Highlight that there are clear indications of affectations (anxiety) to psychological health emotional by vertical and horizontal segregation of gender.

Keywords: gender, anxiety: State-Trait.

\section{Introducción}

Uno de los fines de la Universidad peruana es la de promover y desarrollar la investigación científica en toda la comunidad universitaria tal como lo plantea el inciso $\mathrm{B}$ del artículo segundo de la Ley Universitaria $\mathrm{N}^{\circ} 23733$ y así evitar el fenómeno denominado "techo de cristal" como explica Gonzales \& Junquera (2015) situación invisible que evita que las mujeres desarrollen todo su potencial, es por eso que como diría Jiménez, Murga, Álvarez, Gil, Juan \& Téllez (2006) hay que educar dentro y para la igualdad, ya que este es un principio importante para los educadores que hacen posible la igualdad entre géneros.

Es así como necesitamos que la población universitaria no presente obstáculos para lograr su adecuada participación en actividades de investigación y disminuir los riesgos o problemas de salud mental.
Estos riesgos pueden enmarcarse en las exigencias psicológicas, sociales y académicas, a esto se aúna el estrés propio de la vida universitaria que los conducen a problemas de salud mental como trastornos depresivos, ansiedad, intentos de suicidio, abuso de alcohol y drogas, entre otros (Bohórquez, 2007). Vemos que la importancia de la investigación es identificar niveles de ansiedad como un factor que afecta a la salud integral del estudiante porque influye en la realización de las actividades académicas, de investigación, en sus relaciones interpersonales y familiares llevándolos a menoscabar su desempeño estudiantil en detrimento de su calidad de vida.

La ansiedad es un problema que afecta al ser humano a nivel mundial. Epidemiológicamente se sabe que las mujeres presentan una mayor prevalencia que los varones. Así, Serrano-Barquín, Rojas-García, 
Rugero \& Lopez-Arriaga (2015) sostienen que "aproximadamente $50 / 60$ corresponde a los casos femeninos". Por otro lado, se cree que la un tercio de la población afectada acude a los servicios de salud públicos. Sin embargo podría ser que la población que no acude a buscar ayuda sean hombres (Leal, Barca, Cervera, Vallejo, Giner, \& Cuenca, 2010). Además, hay que tener en cuenta que, en la etapa universitaria, las mujeres se encuentran en edad reproductiva y como mencionan Arenas \& Puigcerver (2009) las mujeres pueden desarrollar problemas ansiosos en edad reproductiva en mayor porcentaje que los hombres, por lo que la presencia de la ansiedad en la etapa universitaria podría tener una connotación de género. En este sentido, Lamas (2000) define la nueva acepción de género como "el conjunto de prácticas, creencias, representaciones y prescripciones sociales que surgen entre los integrantes de un grupo humano en función de una simbolización de la diferencia anatómica entre hombres y mujeres". Ahora, en relación con la ansiedad, Spielberger (2007) la considera como una reacción emocional desagradable frente a un daño anticipado, con características de temor, tensión y que varía en intensidad.

Hay que tener en cuenta que las características biológicas son esenciales y son la base en la definición de nuestra identidad, más los aspectos básicos que nos diferencian es la suma de aspectos geográficos, socio-culturales e institucionalización del entramado social al que se pertenece y así se va definiendo lo masculino y lo femenino como identidades individuales y colectivas. De estas construcciones y valoraciones que se tienen de los hombres y mujeres se van creando un orden social inequitativo. Así, "la dicotomía masculino-femenino, con sus variantes establece estereotipos, las más de las veces rígidos, que condicionan los papeles y limitan las potencialidades humanas de las personas al estimular o reprimir los comportamientos en función de su adecuación al género" (García, 2007, pp. 7-9).

El objetivo general de la investigación fue analizar y relacionar, de forma objetiva, las implicaciones de género y riesgos por predisposición a conducta ansiosa en estudiantes en ciencia y tecnología de la universidad. Para llevarlo a cabo se plantearon como objetivos específicos dos. En primer lugar, identificar los obstáculos en la vida académica, que enfrentan los estudiantes en ciencia - tecnología que podrían predisponer a conductas ansiosas, según el género y segundo, identificar los aspectos favorables y limitantes de la vida personal-familiar en la participación y aportes al conocimiento científico que realizan los estudiantes en ciencia - tecnología, según el género.

Es por eso, por lo que nos planteamos la siguiente hipótesis general, a saber, en la universidad existen brechas de género en la participación y aportes que hacen los estudiantes al desarrollo de la ciencia y tecnología, existiendo desfases con roles de género de la vida personal-familiar los cuales predisponen a conductas ansiosas diferenciada por el género. $Y$ como hipótesis específicas: 1.- Las implicaciones de género como edad, sexo, roles de masculinidad/feminidad, roles de género en vida familiar y personal, predispone a conductas ansiosas. 2.- Los factores favorables como soporte, acceso, participación y aportes de las mujeres estudiantes en grupos de investigación, contribuye hacia la equidad de género. 3.- Es probable distinguir diferencias significativas en conductas ansiosas por áreas de estudio como los son las áreas biomédicas, sociales e ingenierías según el género.

\section{Método}

\section{Participantes}

Participaron estudiantes del 4to. y 5to. año, con matrícula regular 2017 de las 47 escuelas profesionales de las tres áreas académicas de la Universidad: biomédicas, ingenierías y ciencias sociales, para un total de 8,881 estudiantes. Se consideró el 95\% de confianza y un margen de error de $0,5 \%$. La muestra se definió con relación a los estudiantes que están participando en investigaciones de acuerdo con los datos de la UNSA ${ }^{1}$ investiga, constituyendo un total de 368 estudiantes. Los criterios de inclusión fueron: 1) estudiantes con matrícula vigente, 2) que estuvieran realizando investigaciones inscritos en UNSA investiga y 3) qué fueran de ambos sexos. Las personas que participaron de este estudio dieron su consentimiento y lo hicieron de forma voluntaria. En ningún caso se trató de pacientes o mediciones

$1 \quad$ Universidad Nacional de San Agustín, Perú. 
clínicas, sino tener una visión a predisposición de conductas ansiosas condicionadas por la variable de género.

\section{Instrumentos}

Se aplicó de forma conjunta la encuesta de implicaciones de género y el inventario del IDARE, con los cuales se buscó correlacionar e identificar la significancia en la predisposición a conductas ansiosas según variables de género en cuanto a su desarrollo académico y la vida familiar/personal. A la ficha técnica de implicaciones de género se aplicó el proceso de validación y confiabilidad del instrumento que fue evaluado por un experto estadista según método de test-retest. Se hallaron los coeficientes de consistencia interna (alfa de Cronbach entre 0,981 y 0,863$)$.

Por otro lado, el inventario del IDARE que evalúa Ansiedad Rasgo-Estado- se encuentra estandarizado y cuenta con 40 baremos de medición y con tipificación diagnóstica (Spielberger \& DíazGuerrero, 1975).

Finalmente, el procesamiento de datos se realizó en Excel y SPSS con el fin de determinar las correlaciones significativas y prueba estadística de las hipótesis.

\section{Procedimiento}

Se empezó identificando la muestra de estudiantes que eran investigadores, luego se procedió a entregarles los dos instrumentos de evaluación para que fueran respondidos, estando siempre presente un facilitador en el momento de responder las preguntas. La duración de cuestionarios fue en promedio 20 minutos, se ubicó a los participantes en las facultades correspondientes a cada una de las áreas académicas de la Universidad antes indicadas. Luego, de revisión se procedió a subir en las matriz de hojas de Excel por cada instrumento e integrar en SPSS. Al finalizar la investigación, se realizó un taller de presentación de resultados dirigidos a la comunidad universitaria, donde se tuvo participación de estudiantes, algunos docentes y algunos representantes de otras instituciones de la ciudad.

\section{Resultados}

Los estudiantes presentaron edades que oscilaron entre los 19 y 24 años. Las mujeres representaron el $51,93 \%$ y los hombres el $48,07 \%$ de la muestra. Del área de Ciencias Biomédicas se presentó una distribución de mujeres con el $14,9 \%$, y hombres 15,2\%. Del área de las Ingenierías se presentó una distribución de mujeres correspondientes al 32,9\% y hombres $29,6 \%$. Y finalmente, del área de Ciencias Sociales se presentó una distribución de mujeres correspondientes al $4,3 \%$ y de hombres al $3 \%$.

Tabla 1.

Tamaño muestral en media y desviación típica.

Percentil Ansiedad-Estado

\begin{tabular}{llll}
\hline Sexo/Género & N & Media & Desv. típ. \\
\hline Femenino & 192 & 34,49 & 22,799 \\
\hline Masculino & 176 & 32,79 & 23,259 \\
\hline Total & 368 & 33,68 & 23,005 \\
\hline
\end{tabular}

Fuente: elaboración propia.

Luego se presentan los datos significativos de predisposición a conductas ansiosas respecto a la ansiedad-estado.

Figura 1. Porcentaje del nivel de Ansiedad como Rasgo.

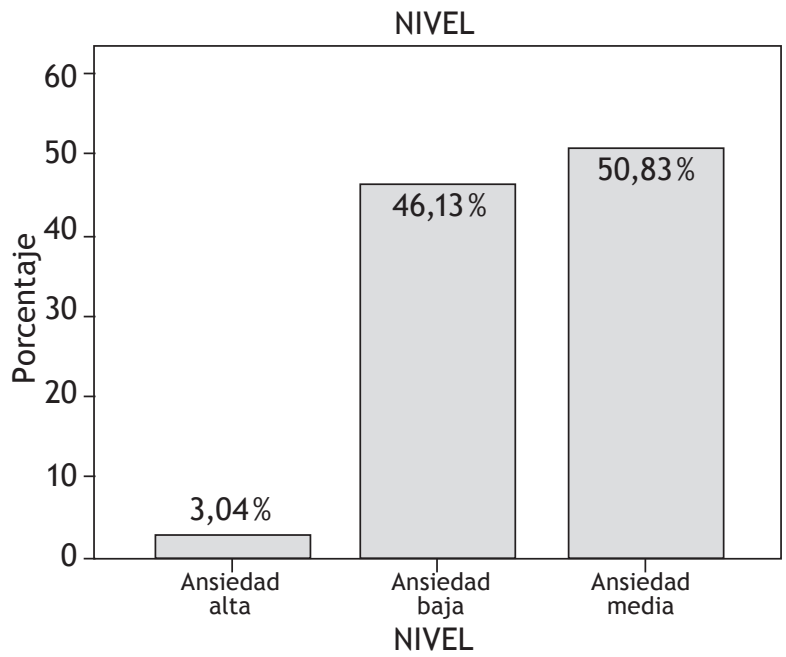

Fuente: elaboración propia. 
Figura 2. Porcentaje del nivel de Ansiedad como Estado.

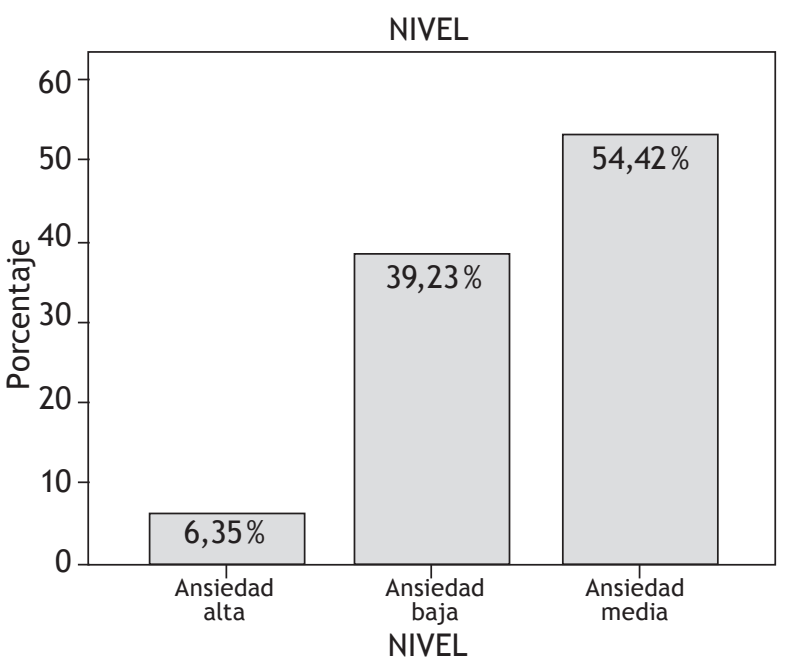

Fuente: elaboración propia.

Los estudiantes presentaron un estado de ansiedad baja y media, tanto a nivel de ansiedad como rasgo y como estado en más del $90 \%$. Es decir, la muestra escogida no presentó una predisposición en sus características de personalidad a desarrollar cuadros ansiosos. En la ansiedad como estado se encontró que la categoría alta está relacionada a los elementos de formación, estudios y cumplimiento de deberes académicos, como se puede observar en las figuras 1 y 2 .

\section{Ansiedad e investigación}

Por otro lado, hay un mayor número de estudiantes mujeres que se dedican a la investigación. Aunque la diferencia fue pequeña con respecto a los hombres fue significativo el resultado, ya que en nuestra población estudiantil hay carreras altamente masculinizadas como por ejemplo todas las relacionadas al área de ingenierías donde los hombres siguen siendo mayoría.

Encontramos ansiedad-estado a nivel moderado en mujeres y en varones. Pero en estos últimos es menos la ansiedad. Con relación a los y las estudiantes que participan en círculos de investigación hay un grupo que presenta ansiedad marcada, siendo mayor en hombres, casi el doble, como se puede apreciar en la figura 3.
Figura 3. Distribución y participación en actividades de investigación y ansiedad.

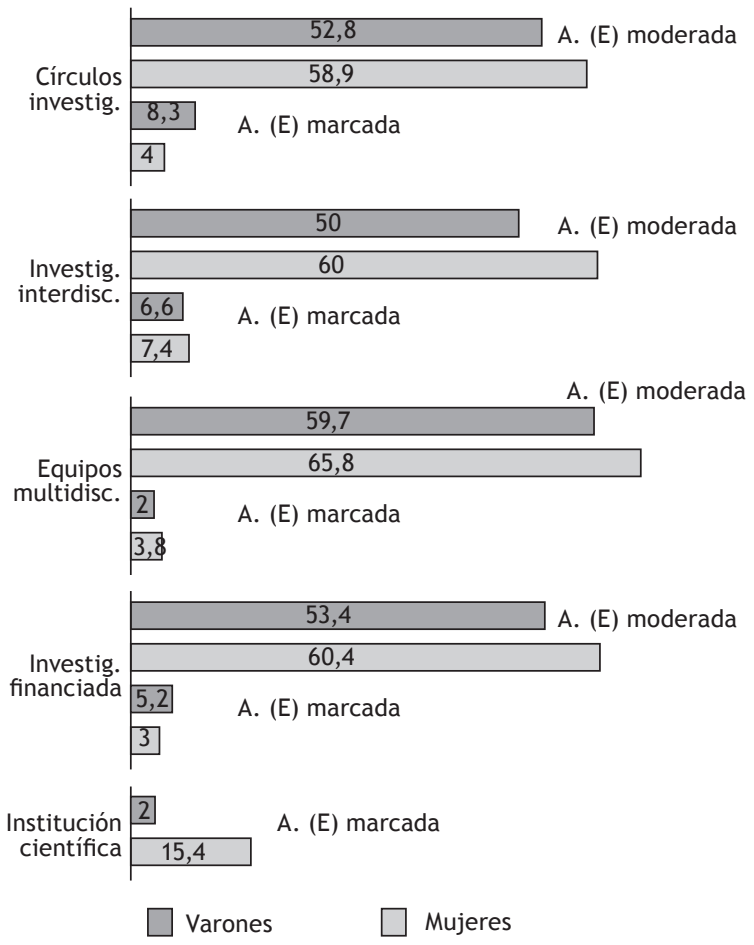

Fuente: elaboración propia.

En cuanto a la participación en investigación interdisciplinaria, se muestra que las mujeres y hombres llegan a un nivel de ansiedad moderada, en mayor cantidad las mujeres y se destaca la categoría de ansiedad marcada con un porcentaje mayor en mujeres que en hombres. La conformación de equipos multidisciplinarios genera en mayor porcentaje una ansiedad moderada en mujeres y en hombres y en cuanto a la ansiedad marcada las mujeres tienen puntuaciones más altas que los hombres.

Respecto a las investigaciones financiadas, se observa que hay una predisposición a la ansiedad estado moderada en mujeres y en hombres es similar la puntuación y en ansiedad marcada los hombres llegan a niveles más elevados que las mujeres. El pertenecer a una institución científica crea ansiedad estado marcada en estudiantes mujeres con una diferencia significativa más alta que en hombres. De las actividades de investigación, la prueba de 
chi-cuadrado muestra valor de 0,846 de significancia de ansiedad en estudiantes mujeres.

\section{Roles familiares y ansiedad}

Los estudiantes que viven solos o con otros familiares - son migrantes - y muestran ansiedad estado marcada de forma muy similar; la diferencia de ansiedad estado marcada es por los roles diferenciados de género en las mujeres que asumen dentro de los grupos familiares, como se observa en la figura 4.

Figura 4. Ansiedad, vida familiar según el género.

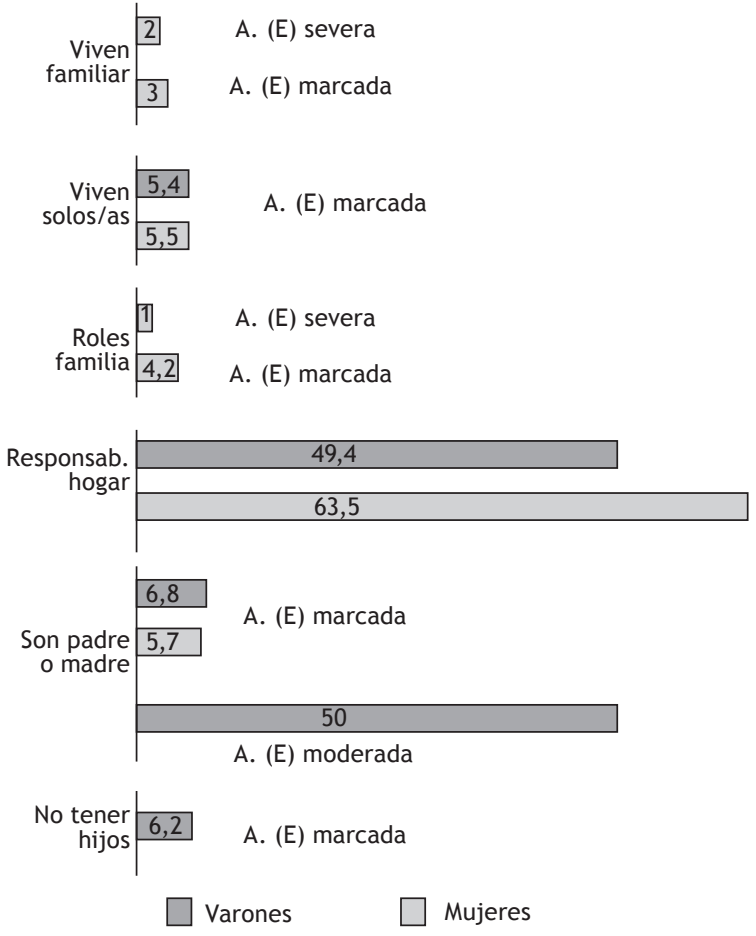

Fuente: elaboración propia.

Respecto de la paternidad y maternidad, los hombres presentan mayor predisposición a conductas ansiosas-estado que las mujeres. Se observa una formación sociocultural sexista y continuidad de roles de las mujeres en las tareas del hogar. En cambio, los hombres reciben mayor apoyo de sus familiares para desarrollar otras actividades fuera del hogar; la responsabilidad económica la asumen más los estudiantes varones; es decir, tienen la función de traer dinero al hogar. Si bien los estudiantes que son padre o madre presentan porcentaje bajo, hay que destacar que el no tener hijos muestra en las estudiantes ansiedad marcada. Éste dato tiene relación con el estado civil de soltera, donde la correlación con conductas ansiosas es alta según el coeficiente $r$ de Pearson es de 0,977 diríamos que hay un anhelo por casarse y ser madres prontamente.

En cuanto al puntaje obtenido en relación a la paternidad se aplicó la prueba de Chi-cuadrado, el valor fue significativo en la ansiedad en los estudiantes $\left(\mathrm{x}^{2}=0,809 ; p<0,05\right)$. Así, se indica una predisposición a conductas ansiosas, lo cual es explica por el proceso de socialización diferencial del género. El vivir solos o con otros familiares por ser migrantes, en ambos géneros, crea predisposición a conductas ansiosas, aplicado el estadístico de Pearson muestra correlación en mujeres es de 0,0774 y en varones de 0,776 valor.

Específicamente, para las mujeres la ansiedad se presenta por asumir roles de apoyo en la crianza de hermanos menores o tareas como la cocina, limpieza en el hogar, es decir, éstas tareas siguen siendo asignadas en las nuevas generaciones de mujeres. La prueba de correlación muestra que la ansiedad es mayor en mujeres con valor 0,859 entre ambas variables.

\section{Ansiedad estado por áreas de estudio}

En el caso de las estudiantes de ingenierías llegan al $63,6 \%$ de ansiedad moderada y ansiedad marcada en el área de Ciencias Sociales llegan a 12,5\% mientras que en hombres la ansiedad moderada es mayor en ingenierías con 55,4\% y ansiedad marcada en Ciencias Sociales con 18,2\%, mientras que en áreas Biomédicas los estudiantes presentan niveles de ansiedad marcada y severa que llegan solo a $5,4 \%$. Hay que puntualizar que el área de Ciencias Sociales muestra cambios en los últimos años según la composición de género, las carreras antes masculinizadas como Sociología, Psicología y Antropología hoy figurativamente tiene rostro femenino.

En relación con la hipótesis de si "es probable distinguir diferencias significativas de niveles en 
conductas ansiosas por áreas: biomédicas, sociales e ingenierías, según el género", se comprueba la misma por las consideraciones antes descritas. Por otro lado, aplicado la prueba de correlación de Pearson se encuentra una correlación significativa de ansiedad en el caso de estudiantes mujeres 0,839 participantes en el estudio, valor que indica que las estudiantes mujeres tienen mayor predisposición a conductas ansiosas que los hombres, con lo cual se puede corroborar la hipótesis de que las mujeres tienden a ser más ansiosas que los varones.

\section{Pruebas de Normalidad de la distribución de la población de estudio}

Respecto a la Ansiedad Estado, la línea en la figura prueba la normalidad y muestra la dispersión y las posiciones variadas en torno a las variables estudiadas de género y ansiedad. Es decir, que las y los estudiantes no tienen un patrón de diferenciación de ansiedad según el género, es variado, no uniforme, según muestra las figuras 5,6 y 7 :

Figura 5. Prueba de normalidad según percentil Ansiedad-Estado.

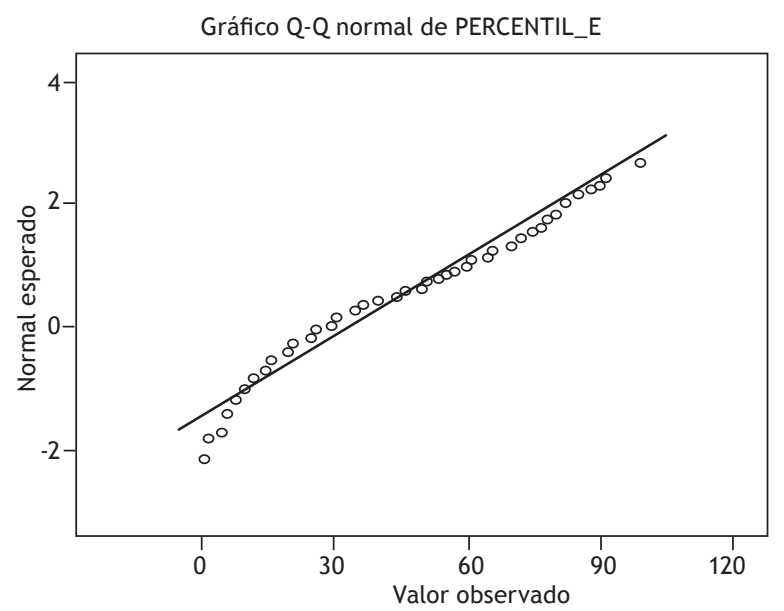

Fuente: elaboración propia.
Figura 6. Prueba de normalidad según percentil Ansiedad-Estado.

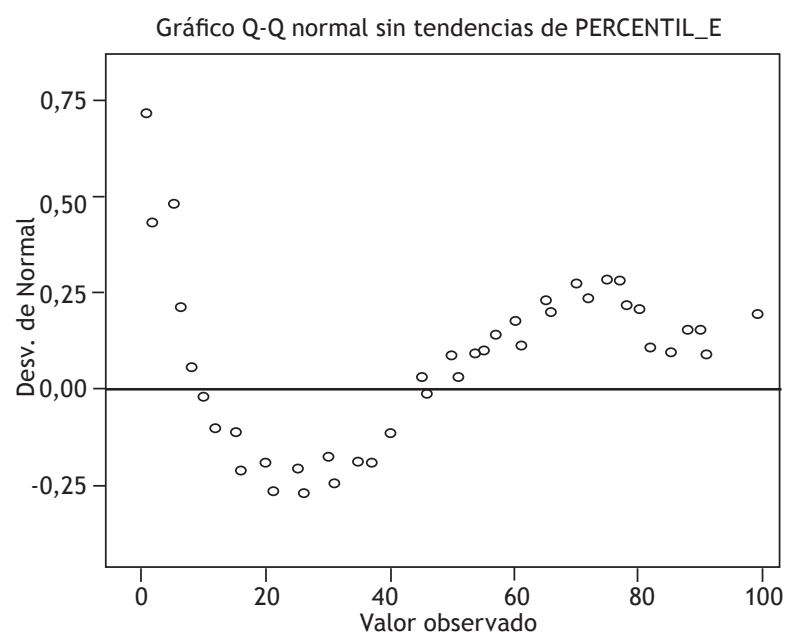

Fuente: elaboración propia.

Figura 7. Prueba de normalidad según percentil de Ansiedad-Estado.

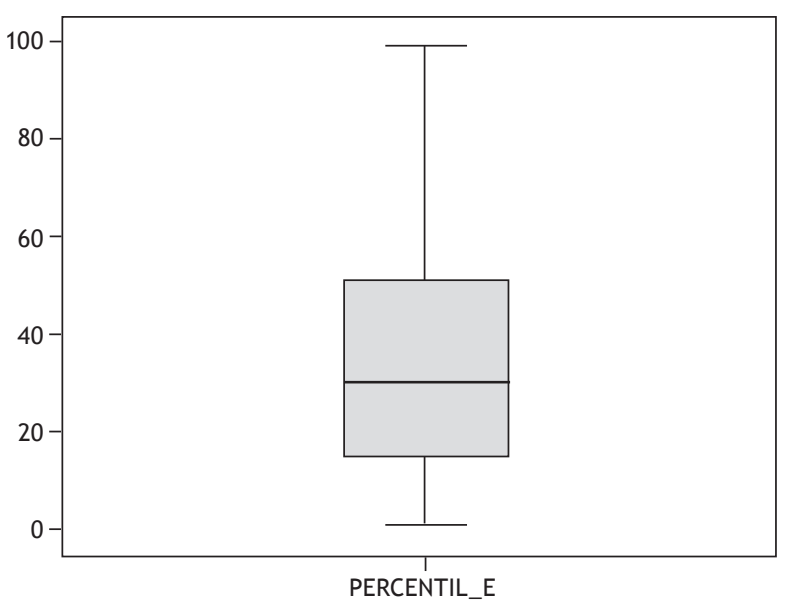

Fuente: elaboración propia. 
Figura 8. Prueba de normalidad según percentil Ansiedad-Rasgo.

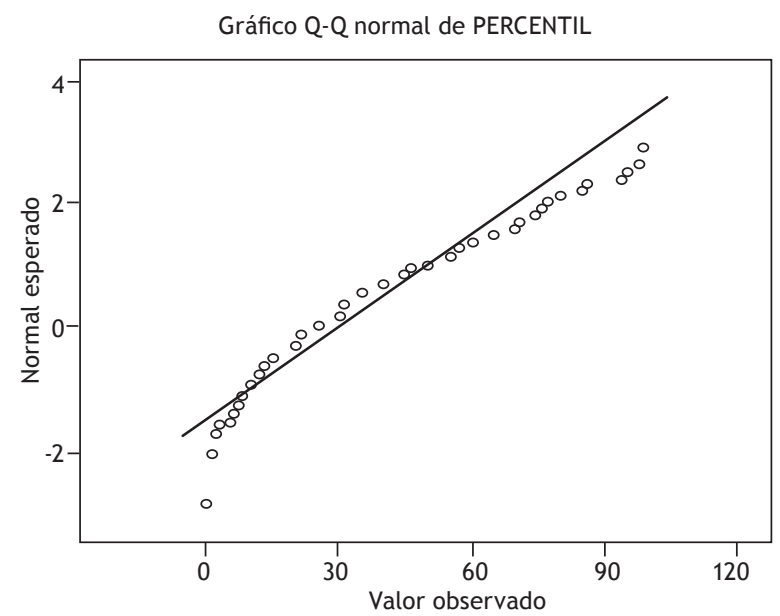

Fuente: elaboración propia.

Figura 9. Prueba de normalidad según percentiles de Ansiedad-Rasgo.

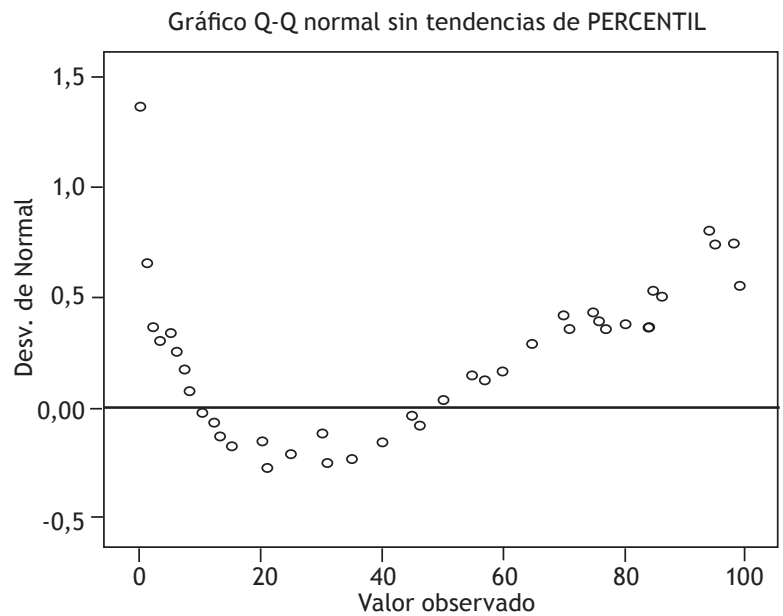

Fuente: elaboración propia.
Figura 10. Prueba de normalidad según percentil Ansiedad-Rasgo.

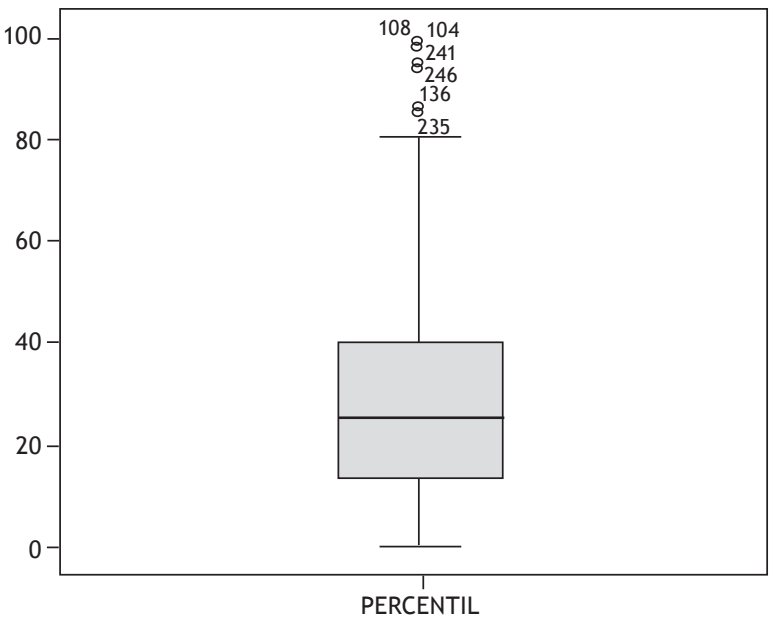

Fuente: elaboración propia.

Las pruebas de normalidad de distribución de la población según Ansiedad-Rasgo, del mismo modo muestran dispersión de datos respecto a la línea de normalidad, según percentil Ansiedad-Rasgo.

\section{Discusión}

Los hallazgos del estudio muestran que las mujeres presentan índices mayores de ansiedad que los hombres. Estos resultados se relacionan con otras investigaciones con base al sexo, como la de Agudelo, Casadiegos \& Sánchez (2008), en donde indican que las mujeres presentan niveles de ansiedad más elevadas que los hombres. También se relacionan con los resultados encontrados entre estudiantes de la Universidad del Norte en Estados Unidos y la Universidad Autónoma del Estado de México (Serrano-Barquín et al., 2015). Las brechas de género y ansiedad entre estudiantes universitarios de ambos sexos se evidencian, sobre todo si lo asociamos con los estereotipos de género que están muy presenten en nuestra sociedad incluso en ámbitos académicos.

En cuanto a la "Ansiedad-Estado" vemos que en su mayoría los estudiantes están dentro de la categoría de "moderada", es decir, pueden manejar la ansiedad generada por las actividades de apoyo en investigación. Sin embargo si lo relacionamos 
con datos obtenidos por Cardona, Pérez, Rivera, Gómez \& Reyes (2014) donde los estudiantes universitarios tienen una "prevalencia de ansiedad de 58 a $55 \%$ en grado leve y 3,0\% moderado” (p. 85), vemos que nuestros porcentajes de ansiedad son más elevados, por lo que los resultados del estudio son significativos y se aproximan a reportes de años anteriores en países como Perú y Brasil donde los encuestados presentan problemas de salud mental y entre ellos de ansiedad. (Weiss Warner, Lyketsos, Frank, Ganzini, \& Carter, 2001).

Aunque las mujeres se desenvuelven dentro de la universidad de forma similar a los hombres, se observa que dentro del hogar siguen desempeñando roles domésticos, asignados por su condición de mujeres hasta en un $60 \%$ mientras que los varones llegan solo al $40 \%$. "Desde la perspectiva de género, esto es a la luz de las normas y expectativas socioculturales acerca de lo que deben ser y hacer los individuos según la categoría sexual que se les asigna al nacer" (Cendales, Vargas-Trujillo, \& Barbosa, 2013, p. 372). Pero también es interesante observar que el porcentaje de varones que cumplen roles domésticos dentro del hogar es significativo, algo más elevado que antes, ya que estas tareas no se le asignaban al estudiante varón.

El estudio muestra que hay más mujeres que se dedican a la investigación que hombres, y de manera especial encontramos con sorpresa que en el área de ingenierías el porcentaje es mayor en mujeres investigadoras, siendo este un espacio dominado tradicionalmente por el sexo masculino. Llama la atención que en el área de las Ciencias Sociales donde por lo general suelen darse mayor participación en actividades o en debates se encuentre que la participación en investigación es baja en ambos sexos.

Es interesante observar que, en el área de Ingeniería hay mayor porcentaje de ansiedad moderada tanto en mujeres como varones, siendo este último grupo que presenta mayor ansiedad. Sin embargo, no encontramos que haya estudios que comparen datos respecto al entorno de participación en ciencia y tecnología en estudiantes. Mientras tanto en el área de biomédicas los puntajes de ansiedad son bajos, llama la atención ya que hay una idea muy marca- da de los altos niveles de exigencia que tienen los estudiantes de esta área dentro de la UNSA. Esto es corroborado en otro estudio que indica que los estudiantes de los primeros semestres de medicina tienen mayores niveles de estrés que los de años superiores, es decir $26,4 \%$ en estudiantes del primer año, y de $8,9 \%$ en el sexto (Celis et al., 2001).

Los estudiantes del área de Ciencias Sociales presentan ansiedad marcada en mayor porcentaje que los estudiantes de ingenierías y áreas biomédicas, pero no tan elevados como lo que encuentra Cardona et al. (2015) donde se reportaron un porcentajes más elevados de ansiedad en estudiantes.

Respecto a las actividades relacionadas con la investigación, tanto en mujeres como hombres, se presentan una ansiedad moderada similar, aunque en las mujeres sea ligeramente mayor pero cuando vemos la categoría marcada los hombres tienen el doble, ( $8 \%$ frente a $4 \%)$ por lo que podríamos asegurar que los hombres suelen ser más ansiosos al momento de desarrollar actividades de investigación.

Por otro lado, respecto a la investigación inter y multidisciplinaria ambos grupos presentan mayor ansiedad al interactuar con otros profesionales para desarrollar actividades de investigación llegando a un 65,8 y $59,7 \%$ en mujeres y hombres respectivamente, siendo mayor en mujeres.

También se determinó que pertenecer a una institución científica genera niveles significativos de ansiedad marcada llegando a $15,4 \%$ en mujeres frente a un bajísimo 2,9\% en hombres. Este dato se puede relacionar con los encontrados en otras instituciones de carácter científico como en la UAEM ${ }^{2}$ en donde, las mujeres presentan mayores síntomas de ansiedad y depresión que los varones (SerranoBarquín et al., 2015).

Los estudiantes que son migrantes y viven con otros familiares tiene menores niveles de ansiedad frente a los que viven solos, tanto en hombres como en mujeres, alcanzando puntajes similares, lo que coincide con el estudio de Riveros, Hernández \& Rivera (2007) quienes señalan que un factor asociado a la ansiedad en estudiantes es la residencia

2 Universidad Autónoma del Estado de México. 
en otros lugares que no sea el hogar. Este mismo grupo de estudiantes que son migrantes y viven con otros familiares desempeñan roles de responsabilidad dentro del hogar, siendo las mujeres las que se sienten más ansiosas frente a los varones. Esto se debe a que aún se sigue asumiendo los roles tradicionales dentro del hogar y son las mujeres las que tiene que cumplir con una serie de responsabilidades que se cumplen todos los días, mientras que los hombres tienen menos responsabilidades y más libertad de poder pasar más tiempo fuera de casa.

\section{Conclusiones}

El estudio es pionero, en nuestra realidad, en relacionar la variable implicaciones de género con la predisposición a conductas ansiosas, aclarando que no se miden datos clínicos. Los resultados indican que los estudiantes investigadores llegan a manifestar niveles de ansiedad promedio dentro de lo esperado. Es decir, dentro de los estándares de normalidad, producto de tareas de investigación y que en su mayoría son mujeres las que desarrollan esta actividad. Frente a la ansiedad-estado se encontró diferencia entre sexos, por lo que se acepta la hipótesis de que las mujeres estudiantes son más ansiosas que los varones a causa de que aún en nuestra sociedad se les sigue asignado actividades o tareas de acuerdo con su sexo que son impuestas por el entorno social.

En relación a las áreas de estudio, en el área de Ciencias Sociales encontramos significativamente que los estudiantes con "ansiedad marcada" y dentro de este grupo los hombres tiene mayor puntuación, de la misma forma que en áreas Biomédicas; mientras que en ingenierías no se presentan problemas de conductas ansiosas tan marcadas, pero si se observa que las estudiantes investigadoras son más ansiosas que los varones, quizás porque su entorno es masculinizado y más competitivo. Resaltar que hay que tener en cuenta que se presentan claros indicios de afectaciones (ansiedad) a la salud psicológica-emocional por segregación vertical y horizontal de género. El estudio permite fundamentar la Implementación del Observatorio de Género y Calidad de Vida de la Universidad.

\section{Agradecimientos}

A nuestra Universidad por el financiamiento otorgado para el desarrollo de la investigación, a todos los integrantes de apoyo de la investigación y a los participantes por su invaluable información y tiempo de calidad.

\section{Referencias}

Agudelo, D., Casadiegos, C., \& Sánchez, D. (2008). Características de ansiedad y depresión en estudiantes universitarios. International Journal of Psychological Research, 1(1), 34-39.

Arenas, C. \& Puigcerver, A. (2009) Diferencias entre hombres y mujeres en los trastornos de ansiedad: una aproximación psicobiológica. Escritos de Psicología, 3(1), 20-29.

Bohórquez, A. (2007). Prevalencia de depresión y ansiedad según las escalas de Zung y evaluación de la asociación con el desempeño académico en los estudiantes de medicina de la Pontificia Universidad Javeriana. Bogotá. (Tesis de grado). Pontificia Universidad Javeriana. http:// www.javeriana.edu.co/biblos/tesis/medicina/tesis37.pdf

Cardona-Arias, J., Pérez-Restrepo, D., RiveraOcampo, S., Gómez-Martínez, J., \& Reyes, A. (2015) Prevalencia de ansiedad en estudiantes universitarios. Diversitas: Perspectivas en Psicología, 11(1), 79-89. Doi: https://doi. org/10.15332/s1794-9998.2015.0001.05

Celis, J., Bustamante, M., Cabrera, D., Cabrera, M., Alarcón W., \& Monje, E. (2001) Ansiedad y estrés académico en estudiantes de Medicina Humana del primer y Sexto Año. Anales de la Facultad de Medicina, 62(1), 25-30. Doi: https:// doi.org/10.15381/anales.v62i1.4143

Cendales, B., Vargas-Trujillo, E., \& Barbosa, C. (2013). Factores psicológicos asociados al desempeño académico en los cursos universitarios de estadística: diferencias por sexo y área de titulación. Avances en Psicología Latinoamericana, 31(2), 363-375. 
García, M. (2007). ABC de género en la administración pública. Instituto Nacional de las Mujeres/ Programa de las Naciones Unidas para el Desarrollo, México, pp. 7-9. Recuperado de: http://cedoc.inmujeres.gob.mx/documentos_ download/100903.pdf

Gonzales, A. \& Junquera, B. (2015) El techo de cristal. Oviedo: Universidad de Oviedo.

Jiménez, C., Murga M., Álvarez B., Gil, J., \& Téllez, J. (2006) Estudiantes universitarios ante la cuestión de género: estudio propedéutico para la intervención pedagógica. Ensaio Avaliação e Políticas Públicas em Educação, 14(53), 437-456. Doi: https://doi.org/10.1590/ S0104-40362006000400003

Lamas, M. (2000). Diferencias de sexo, género y diferencia sexual. Cuicuilco, 7(18), (enero-abril).

Leal, C., Barca, E., Cervera, S., Vallejo, J. Giner, J., \& Cuenca, E. (2010). Trastornos Neuróticos. Barcelona, España. Lexus, 4(8), 99-114.

Riveros, M., Hernández, H., \& Rivera, J., (2007) Niveles de depresión y ansiedad en estudiantes universitaria de Lima metropolitana. Revista IIPSI Facultad de psicología UNMSM, 10(1), 91-102.
Serrano-Barquín, C., Rojas-García, A., Ruggero, C., \& López-Arriaga, M. (2015) Depresión y ansiedad desde los estudios de género en estudiantes Universitarios. Revista de Psicología de la Universidad del Estado de México, 4(8), 99-114.

Spielberger, C. (2007). La naturaleza de la ansiedad y su medida de investigación transcultural. Revista de Psicología de la Universidad Nacional Mayor de San Marcos, 9(1), 83-96.

Spielberger, C. D. \& Díaz-Guerrero, R. (1975). IDARE Inventario de Ansiedad: Rasgo-Estado. Manual e instructivo. México: El Manual Moderno S. A.

Vargas-Trujillo, E. \& Gambara, H. (2008) Evaluación de programas y proyectos de intervención: una guía con enfoque de género. (1ra. ed.) Colombia: Bogotá D. C.

Weiss, L., Warner, T., Lyketsos, C., Frank, E., Ganzini, L., \& Carter D. (2001). Perceptions of academic vulnerability associated with personal illness: A study of 1027 students at nine medical schools. Comprehensive Psychiatry, 42(1), 1-15. Doi: https://doi.org/10.1053/comp.2001.19747 